\title{
Outcomes associated with planned place of birth among women with low-risk pregnancies
}

\author{
Eileen K. Hutton PhD, Adriana Cappelletti BHSc, Angela H. Reitsma RM MSc, Julia Simioni MSc, \\ Jordyn Horne BSc, Caroline McGregor BSc, Rashid J. Ahmed BSC
}

Competing interests: None declared.

This article has been peer reviewed.

Accepted: Oct. 26, 2015

Online: Dec. 21, 2015

Correspondence to:

Eileen Hutton,

huttone@mcmaster.ca

CMAJ 2016. DOI:10.1503/ cmaj.150564

\begin{abstract}
Background: Previous studies have shown that planned home birth is associated with a decreased likelihood of intrapartum intervention with no difference in neonatal outcomes compared with planned hospital birth. The purpose of our study was to evaluate different birth settings by comparing neonatal mortality, morbidity and rates of birth interventions between planned home and planned hospital births in Ontario, Canada.
\end{abstract}

Methods: We used a provincial database of all midwifery-booked pregnancies between 2006 and 2009 to compare women who planned home birth at the onset of labour to a matched cohort of women with low-risk pregnancies who had planned hospital births attended by midwives. We conducted subgroup analyses by parity. Our primary outcome was stillbirth, neonatal death $(<28 \mathrm{~d})$ or serious morbidity (Apgar score $<4$ at 5 min or resuscitation with positive pressure ventilation and cardiac compressions).

Results: We compared 11493 planned home births and 11493 planned hospital births. The risk of our primary outcome did not differ significantly by planned place of birth (relative risk [RR] 1.03, 95\% confidence interval [CI] 0.68-1.55). These findings held true for both nulliparous (RR 1.04, 95\% Cl 0.62-1.73) and multiparous women (RR 1.00, 95\% Cl 0.49-2.05). All intrapartum interventions were lower among planned home births.

Interpretation: Compared with planned hospital birth, planned home birth attended by midwives in a jurisdiction where home birth is well-integrated into the health care system was not associated with a difference in serious adverse neonatal outcomes but was associated with fewer intrapartum interventions.
I n Ontario, Canada, the College of Midwives of Ontario has regulated midwifery since 1994, and increasing numbers of women with low obstetrical risk and their newborns receive care in a publicly funded, midwifery-led continuity of care model. ${ }^{1}$ Midwives have admission and discharge privileges at their local hospitals and are able to consult or transfer care to other health care providers if required. In Ontario, midwives attend a small proportion of all births in the province (10\%), and about $20 \%$ of the births they attend take place at home. ${ }^{2}$ A comprehensive record is maintained for every woman and infant in a midwife's care. Until 2009, this record was submitted to the provincial Ministry of Health and Long-term Care (MOHLTC) through the Ontario Midwifery Program to access reimbursement for care provided.

In the last century, Western culture has come to view hospital birth as safer than home birth. ${ }^{3}$ Recently, however, the value of hospital birth for all women with low-risk pregnancies has come into question; it has been suggested that in the absence of benefit, a planned hospital birth for this population may increase the use of intrapartum interventions, including cesarean delivery. ${ }^{4-7}$ Even though recent studies comparing planned home and hospital births have had moderate sample sizes, they are individually limited in their ability to report definitively on rare outcomes such as death. Owing to a lack of evidence from randomized controlled trials (RCTs) to show that restricting a woman's freedom to choose a place of birth prevents harm, the authors of a 2012 Cochrane review of planned hospital versus planned home births concluded that home birth services with collaborative medical backup should be established and offered to women with low-risk pregnancies in all jurisdictions. ${ }^{8}$ This conclusion, along with findings from the large English Birthplace Cohort Study, ${ }^{4}$ may be what prompted the National Institute for Health and Care Excellence (NICE) 
in England to update its intrapartum care guidelines to recommend that, for women at low risk of birth-associated complications, home birth should be considered a generally safe option. ${ }^{9}$ With the paucity of information derived from RCTs, ${ }^{8}$ observational studies are essential to continue to inform and monitor maternal and infant outcomes for women at low obstetrical risk who plan home or hospital birth, and to continue to provide pregnant women with quality information about choice of birthplace.

The primary purpose of this retrospective cohort study was to determine the risk of stillbirth or neonatal death or serious neonatal morbidity among women at low obstetrical risk whose deliveries were attended by midwives and who had planned a home birth at the onset of labour, compared with women at low obstetrical risk who planned a hospital birth at the onset of labour. In addition, we also compared the incidence of maternal death and morbidity, birth interventions and breastfeeding between planned home births and planned hospital births.

\section{Methods}

\section{Study design and setting}

We conducted a retrospective cohort study using maternal and infant data from midwiferybooked pregnancies invoiced between Mar. 31, 2006, and Apr. 1, 2009, and collected by the Ontario Midwifery Program of the Ontario MOHLTC. Registered midwives provided care to all women involved in the study. The study was approved by the Hamilton Health Sciences Research Ethics Board.

\section{Study participants}

From all midwifery-booked pregnancies in the data set, we excluded women who had miscarriages or abortions and women who were discharged from care during pregnancy or who experienced fetal death before labour. We also excluded records that had missing values for variables required for matching (parity or number of previous cesarean deliveries), exposure classification (planned place of birth) or mortality outcome assessment (stillbirth, neonatal death or whether fetal death occurred before or during labour).

Records were classified as planned home or planned hospital births using a data field that collected the intended place of birth at the onset of labour. Thus, adverse outcomes among women and infants who began intrapartum care at home, but who were transferred to hospital during labour, were captured in the home birth group and risk of bias in favour of home birth was avoided. In the planned home birth group, we attempted to capture all records of women who intended a home birth at the onset of labour by including all records indicating "home," "undecided" or "other" as the intended place of birth at the onset of labour, because women with the latter 2 designations may include home births.

Logic checks were used to detect possible coding errors in the planned place of birth data field. If a record indicated a contraindication to home birth (e.g., preterm birth, breech birth, multiple gestation), an intervention that was inconsistent with home birth (e.g., oxytocin induction of labour) or an antenatal transfer of care to a physician, 2 experienced midwives reviewed the record, blinded to outcome. An algorithm used in previous work ${ }^{5}$ was employed to determine whether to include the record in the primary analyses, to permanently exclude the record or to maintain the record for the sensitivity analyses (Figure 1 and Appendix 1, available at www.cmaj.ca/lookup/suppl/doi:10.1503/ cmaj.150564/-/DC1).

A comparison cohort was formed from all records that indicated a planned hospital birth at the onset of labour. To ensure that the comparison group was similarly low risk to that of the planned home birth group, we excluded records that reported a condition or complication that requires mandatory antenatal consultation or transfer of care to a physician according to the College of Midwives of Ontario. ${ }^{10}$ Thus, we excluded records that indicated alcohol or drug dependency, chronic hypertension, type 1 diabetes, a heart condition, hepatitis B, HIV, isoimmunization, anemia unresponsive to therapy, antepartum bleeding, eclampsia, gestational diabetes, intrauterine growth restriction or small for gestational age, oligohydramnios, placenta previa, placental abruption, polyhydramnios or pregnancy-induced hypertension. We also excluded records if they indicated any of the following conditions: preterm delivery $(<37 \mathrm{wk}$ gestation), breech presentation at birth, more than one previous cesarean delivery, multiple pregnancy or medical induction (with oxytocin or prostaglandin). We did not exclude postterm pregnancies from either group.

Women with a single previous lower segment incision are not excluded from home birth in Ontario, ${ }^{11}$ but along with first-time mothers, may be at increased risk of obstetric complication. ${ }^{12}$ An imbalance in these potential confounding factors between groups could result in an overestimate of adverse events in the cohort with a greater proportion of first-time mothers or women with a previous uterine scar. Therefore, we stratified eligible records on parity ( 0 and $\geq 1)$ and on previous cesarean delivery ( 0 or 1$)$. 
We used frequency matching of all eligible planned home birth records to a random sample of eligible planned hospital birth records based on parity and prior cesarean delivery in a 1:1 ratio. We planned to examine demographic and other prognostic characteristics (presented in Table 1) for clinically important differences that would require further adjusting.

\section{Outcomes}

Our primary outcome was a composite measure of stillbirth, neonatal death or serious morbidity. Any record that indicated one or more component event was considered to have had the primary outcome. We defined stillbirth as any fetal death after the onset of labour, and neonatal death as death of the infant within 28 days of birth. We excluded deaths associated with lethal anomalies from the primary outcome. Two authors, blinded to group, independently reviewed stillbirths or neonatal deaths that indicated a congenital anomaly, and a consensus decision was made regarding exclusion from the primary outcome. The composite outcome included the following measures of neonatal morbidity: ${ }^{1}$ Apgar score below 4 at 5 minutes and infant resuscitation requiring positive pressure ventilation (PPV) and cardiac compressions. ${ }^{2}$ We compared groups on stillbirth, perinatal death (defined as stillbirth after the onset of labour or death within 7 days of birth),

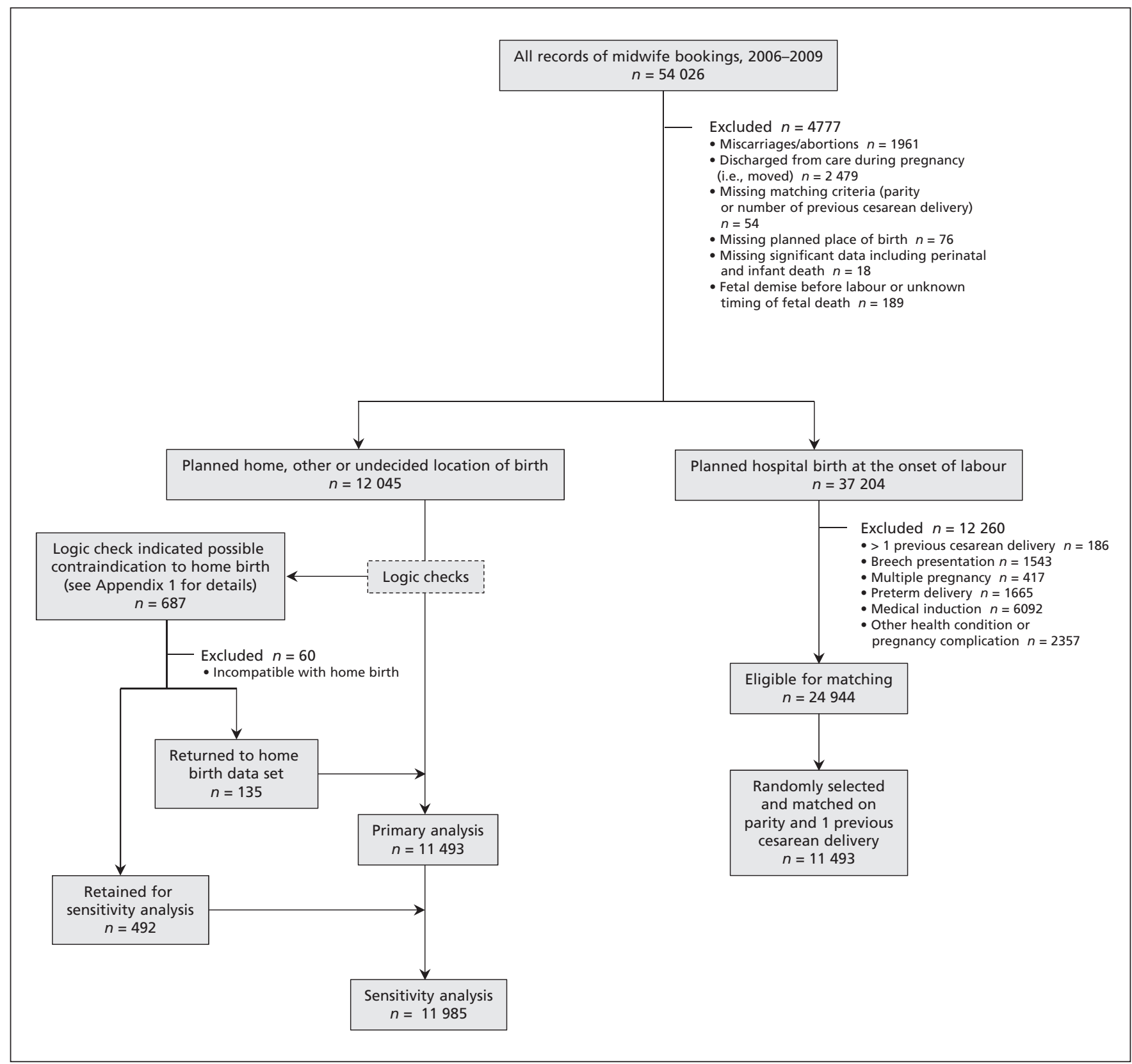

Figure 1: Selection of the study groups. 
and neonatal death excluding lethal anomalies. The risk of any perinatal or neonatal death including lethal anomalies was also compared between groups.

Our secondary outcomes included intrapartum interventions, maternal morbidity, maternal death, breastfeeding and infant birth weight less than 2500 g. We examined birth weight less than $2500 \mathrm{~g}$ as a marker for home births that may have been inappropriately planned owing to a lack of effective screening during pregnancy. The frequency of intrapartum interventions was compared between groups, including augmentation, pharmaceutical pain relief, episiotomy, assisted vaginal delivery and cesarean delivery. We compared the risk of maternal morbidity between planned home and planned hospital births including postpartum hemorrhage and severe perineal trauma. Postpartum hemorrhage was listed as a complication on the Ontario Midwifery Program data form but was not defined further. Typically, midwives classify women as having a postpartum hemorrhage based on estimated blood loss greater than $1000 \mathrm{~mL}$, symptoms or required level of intervention. Severe perineal trauma was defined as 3rd or 4th degree perineal laceration. The frequency of maternal death due to direct obstetric cause was reported among planned home and planned hospital births. Exclusive breastfeeding was compared between groups at 3 and 10 days postpartum. We reported the frequency of an Apgar score less than 4 at 1 minute, Apgar score less than 7 at 5 minutes, significant congenital anomalies, actual place of birth and emergency medical services called to the home during or immediately after the birth between the 2 comparison groups.

\section{Statistical analyses}

Demographic and prognostic baseline characteristics were calculated for each group. Continuous variables were categorized based on clinically relevant cut-offs. All data were presented as counts and percentages. We calculated Pearson $\chi^{2}$ with Yates continuity correction, relative risk (RR) and $95 \%$ confidence intervals (CIs) for all prespecified comparative analyses. We have reported the number of missing values for all outcomes and characteristics that had missing values. All data preparation and statistical analyses were done using IBM SPSS Statistics $22^{13}$ and $\mathrm{R}$ statistical software version 2.14.1. ${ }^{14}$

To detect differences by parity, we planned a priori to perform subgroup analyses of first-time pregnancies and subsequent pregnancies for all primary and secondary outcomes in both the primary analysis and the sensitivity analysis.

We conducted a meta-analysis to pool the results from the study reported here (2006-2009)

Table 1: Baseline characteristics of women planning home or hospital birth

\begin{tabular}{|ccc|}
\hline & \multicolumn{2}{c|}{ No. (\%) } \\
\cline { 2 - 3 } Characteristic & $\begin{array}{c}\text { Planned home } \\
(n=11493)\end{array}$ & $\begin{array}{c}\text { Planned hospital } \\
(n=11493)\end{array}$ \\
\hline
\end{tabular}

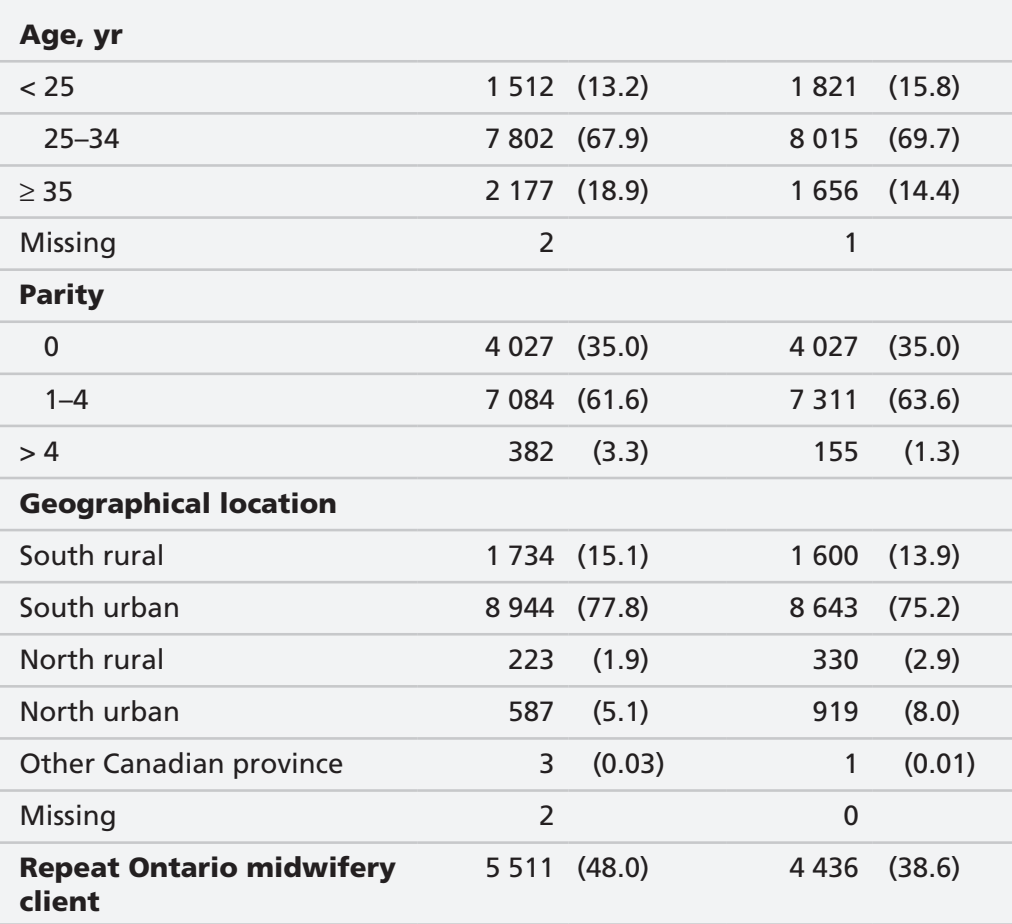

$\begin{array}{lll}\text { Missing } & 2 & 0\end{array}$

Previous cesarean

deliveries

\begin{tabular}{|c|c|c|c|c|}
\hline 0 & 11206 & $(97.5)$ & 11206 & $(97.5)$ \\
\hline 1 & 271 & (2.4) & 287 & (2.5) \\
\hline$>1$ & 16 & $(0.1)$ & 0 & $(0.0)$ \\
\hline Maternal smoking & 477 & (4.3) & 647 & (5.8) \\
\hline Missing & 159 & & 156 & \\
\hline Aboriginal & 217 & (2.1) & 225 & (2.1) \\
\hline Missing & 1088 & & 968 & \\
\hline \multicolumn{5}{|l|}{$\begin{array}{l}\text { Gestational age at } \\
\text { booking }\end{array}$} \\
\hline$<12 w k, 6 d$ & 7910 & $(68.8)$ & 7522 & $(65.4)$ \\
\hline$\geq 13 w k$ to $<27 w k, 6 d$ & 2868 & $(25.0)$ & 3295 & $(28.7)$ \\
\hline$\geq 28 w k$ & 715 & $(6.2)$ & 676 & (5.9) \\
\hline Multiple birth & 2 & $(0.02)$ & 0 & $(0.0)$ \\
\hline Breech presentation & 35 & $(0.3)$ & 0 & $(0.0)$ \\
\hline \multicolumn{5}{|l|}{ Gestational age } \\
\hline$<37$ wk & 101 & $(0.9)$ & 0 & $(0.0)$ \\
\hline $37 w k-41 w k, 6 d$ & 11210 & $(97.5)$ & 11414 & $(99.3)$ \\
\hline$>41 \mathrm{wk}, 6 \mathrm{~d}$ & 182 & $(1.6)$ & 79 & $(0.7)$ \\
\hline
\end{tabular}


and our previous study that used data collected between 2003 and 2006. Owing to changes in data collection since the earlier study, our primary composite outcome has changed. Therefore, we calculated the incidence of stillbirth or neonatal death excluding lethal anomalies per 1000 births and 95\% CIs and an RR and 95\% CI for the entire population, as well as stratified by parity, from both studies.

\section{Results}

\section{Selection of the cohort}

Between Mar. 31, 2006, and Apr. 1, 2009, we found 54026 records of midwifery-booked pregnancies invoiced to the MOHLTC. After excluding ineligible records (Figure 1 and Appendix 1), 11493 planned hospital birth records were matched to 11493 planned home birth records on

Table 2 (part 1 of 2): Comparison of intrapartum variables and postpartum maternal variables with relative risk presented for selected outcomes

\begin{tabular}{|c|c|c|c|c|c|}
\hline \multirow{2}{*}{$\begin{array}{l}\text { Variable } \\
\text { Labour augmentation }\end{array}$} & \multicolumn{2}{|c|}{$\begin{array}{l}\text { Planned home, no. (\%) } \\
\qquad n=11493\end{array}$} & \multicolumn{2}{|c|}{$\begin{array}{l}\text { Planned hospital, no. (\%) } \\
\qquad n=11493\end{array}$} & \multirow{2}{*}{$\frac{\mathrm{RR}(95 \% \mathrm{Cl})}{0.79(0.76-0.82)}$} \\
\hline & 3570 & $(31.1)$ & 4438 & (38.7) & \\
\hline Nulliparous* & 1605 & $(40.9)$ & 1927 & $(49.2)$ & $0.83(0.79-0.87)$ \\
\hline Missing & 105 & & 114 & & \\
\hline Multiparous* & 1965 & $(27.1)$ & 2511 & (35.5) & $0.76(0.73-0.80)$ \\
\hline Missing & 210 & & 393 & & \\
\hline \multicolumn{6}{|l|}{ Method of labour augmentation } \\
\hline Amniotomy & 2896 & $(25.2)$ & 3501 & $(30.5)$ & \\
\hline Oxytocin & 1000 & (8.7) & 1551 & $(13.5)$ & \\
\hline Prostaglandin & 3 & $(0.03)$ & 12 & $(0.1)$ & \\
\hline Missing method of augmentation & 14 & & 21 & & \\
\hline Pharmaceutical pain relief & 1866 & $(16.2)$ & 4825 & $(42.0)$ & $0.39(0.37-0.41)$ \\
\hline Nulliparous* & 1388 & (34.5) & 2413 & $(59.9)$ & $0.58(0.55-0.60)$ \\
\hline Multiparous* & 478 & $(6.4)$ & 2413 & $(32.3)$ & $0.20(0.18-0.22)$ \\
\hline \multicolumn{6}{|l|}{ Type of pharmaceutical pain relief } \\
\hline Nitrous oxide & 465 & (4.1) & 1938 & $(16.9)$ & \\
\hline Narcotic analgesia & 187 & $(1.6)$ & 660 & $(5.7)$ & \\
\hline Epidural & 1221 & $(10.6)$ & 2608 & $(22.7)$ & \\
\hline Spinal & 259 & $(2.3)$ & 417 & (3.6) & \\
\hline Pudendal & 17 & $(0.1)$ & 34 & $(0.3)$ & \\
\hline General anesthesia & 40 & $(0.3)$ & 59 & $(0.5)$ & \\
\hline \multicolumn{6}{|l|}{ Mode of birth } \\
\hline Spontaneous vaginal & 10449 & $(90.9)$ & 9994 & $(87.0)$ & \\
\hline Assisted vaginalt & 370 & $(3.2)$ & 591 & (5.1) & $0.61(0.54-0.70)$ \\
\hline Nulliparous* & 313 & $(8.9)$ & 452 & $(13.2)$ & $0.68(0.59-0.78)$ \\
\hline Multiparous* & 57 & $(0.8)$ & 139 & $(1.9)$ & $0.40(0.30-0.55)$ \\
\hline \multicolumn{6}{|l|}{ Method of assisted vaginal birth } \\
\hline Forceps & 125 & $(1.1)$ & 194 & $(1.7)$ & \\
\hline Vacuum & 223 & (1.9) & 376 & (3.3) & \\
\hline Forceps and vacuum & 22 & $(0.2)$ & 21 & $(0.2)$ & \\
\hline Cesarean delivery $¥$ & 672 & (5.8) & 903 & (7.9) & $0.74(0.67-0.82)$ \\
\hline Nulliparous* & 531 & $(13.2)$ & 611 & $(15.2)$ & $0.87(0.78-0.97)$ \\
\hline Multiparous* & 141 & $(1.9)$ & 292 & (3.9) & $0.48(0.40-0.59)$ \\
\hline Missing mode of birth§ & 2 & $(0.02)$ & 5 & $(0.04)$ & \\
\hline
\end{tabular}


parity and previous cesarean delivery. An additional 492 records were included in the planned home birth group for the sensitivity analysis. Thus, the primary analysis included 22986 records and the sensitivity analysis included 23478 records.

\section{Description of the cohort}

Members of the planned home and planned hospital groups showed similar baseline characteristics (Table 1). First-time mothers represented $35.0 \%$ of women in both groups. We did not find

Table 2 (part $\mathbf{2}$ of 2): Comparison of intrapartum variables and postpartum maternal variables with relative risk presented for selected outcomes

\begin{tabular}{|c|c|c|c|c|}
\hline Variable & $\begin{array}{l}\text { Planned ho } \\
\qquad n=1\end{array}$ & $\begin{array}{l}\text { me, no. (\%) } \\
1493\end{array}$ & $\begin{array}{l}\text { Planned hospital, no. (\%) } \\
\qquad n=11493\end{array}$ & $\mathrm{RR}(95 \% \mathrm{Cl})$ \\
\hline \multicolumn{5}{|l|}{ Laceration } \\
\hline Any laceration & 6057 & $(52.7)$ & $6785 \quad(59.0)$ & \\
\hline First-degree perineal & 1966 & $(17.1)$ & $2067 \quad(18.0)$ & \\
\hline Second-degree perineal & 3089 & $(26.9)$ & $3637 \quad(31.6)$ & \\
\hline Third-degree perineal & 118 & $(1.0)$ & $230 \quad(2.0)$ & \\
\hline Fourth-degree perineal & 33 & $(0.3)$ & $37 \quad(0.3)$ & \\
\hline Labial & 831 & (7.2) & $684 \quad(5.9)$ & \\
\hline Vaginal & 1041 & (9.1) & $1198 \quad(10.4)$ & \\
\hline Cervical & 4 & $(0.03)$ & $(0.1)$ & \\
\hline $\begin{array}{l}\text { Severe perineal trauma (third or fourth } \\
\text { degree perineal laceration) }\end{array}$ & 151 & $(1.3)$ & $266 \quad(2.3)$ & $0.57(0.47-0.69)$ \\
\hline Nulliparous* & 126 & $(3.1)$ & $189 \quad(4.7)$ & $0.67(0.53-0.83)$ \\
\hline Multiparous* & 25 & $(0.3)$ & $77 \quad(1.0)$ & $0.33(0.21-0.51)$ \\
\hline Episiotomy & 464 & $(4.0)$ & $680 \quad(5.9)$ & $0.68(0.61-0.77)$ \\
\hline Nulliparous* & 373 & $(9.3)$ & $510 \quad(12.7)$ & $0.73(0.65-0.83)$ \\
\hline Missing & 4 & & 3 & \\
\hline Multiparous* & 91 & $(1.2)$ & $(2.3)$ & $0.54(0.42-0.69)$ \\
\hline Missing & 7 & & 6 & \\
\hline Postpartum hemorrhage & 286 & $(2.5)$ & $348 \quad(3.0)$ & $0.82(0.70-0.96)$ \\
\hline Nulliparous* & 130 & (3.2) & $146 \quad(3.6)$ & $0.89(0.71-1.12)$ \\
\hline Multiparous* & 156 & (2.1) & 202 & $0.77(0.63-0.95)$ \\
\hline Any maternal mortality & 0 & (0) & $0 \quad(0)$ & \\
\hline \multicolumn{5}{|l|}{ Actual place of birth } \\
\hline Home & 8588 & $(74.7)$ & $344 \quad(3.0)$ & \\
\hline Nulliparous* & 2189 & $(54.4)$ & $133 \quad(3.3)$ & \\
\hline Multiparous* & 6399 & $(85.7)$ & $211 \quad(2.8)$ & \\
\hline Hospital & 2810 & $(24.4)$ & 11118 (96.7) & \\
\hline Other & 95 & $(0.8)$ & $(0.3)$ & \\
\hline $\begin{array}{l}\text { Emergency services called to home during } \\
\text { or immediately after birth }\end{array}$ & 927 & $(8.8)$ & $190 \quad(1.7)$ & \\
\hline Nulliparous* & 341 & (8.5) & $(1.3)$ & \\
\hline Missing & 17 & & 97 & \\
\hline Multiparous* & 586 & (7.9) & (1.9) & \\
\hline Missing & 35 & & 36 & \\
\hline \multicolumn{5}{|c|}{$\begin{array}{l}\text { Note: Denominator has been adjusted to exclude missing data. } \mathrm{Cl}=\text { confidence interval, RR = relative risk. } \\
\text { *Nulliparous } n=4027 \text {; multiparous } n=7466 \text {. } \\
\text { tRelative risk of assisted vaginal birth compared with spontaneous vaginal birth among all vaginal births. Denominators were as follows: nulliparous planned } \\
\text { home births } n=3496 \text {, multiparous planned home births } n=7323, \text { nulliparous planned hospital births } n=3416 \text {, multiparous planned hospital births } n=7169 \text {. } \\
\text { †Relative risk of cesarean delivery compared with vaginal birth among all records with mode of birth indicated. } \\
\text { §Seven records were missing mode of birth ( } 2 \text { planned home births and } 5 \text { planned hospital births). All } 7 \text { records were from multiparous mothers. }\end{array}$} \\
\hline
\end{tabular}


a clinically important difference in smoking, maternal age or postmaturity that required adjustment. Slightly fewer women smoked in the planned home birth group (4.3\%) compared with the planned hospital birth group (5.8\%).

\section{Outcomes}

Of 11493 women in each group, $74.7 \%$ of those in the planned home birth group gave birth at home and $96.7 \%$ of those in the planned hospital group gave birth in hospital (Table 2). Members in both groups required emergency medical services during or immediately after birth: $8.1 \%$ in the planned home birth group and $1.7 \%$ in the planned hospital birth group.

Compared with women planning hospital births, women who planned home births were less likely to undergo labour augmentation (RR $0.79,95 \%$ CI $0.76-0.82)$ or to receive pharmaceutical pain relief (RR $0.39,95 \%$ CI $0.37-0.41$ ) (Table 2). Women in both groups had a high likelihood of having a spontaneous vaginal birth (90.9\% for the planned home birth group and $87.0 \%$ for the planned hospital birth group). Compared with the planned hospital birth group, women in the planned home birth group had fewer assisted vaginal deliveries (RR 0.61, 95\% CI 0.54-0.70) and fewer cesarean deliveries (RR $0.74,95 \%$ CI $0.67-0.82$ ), and were less likely to experience severe perineal trauma (RR 0.57, 95\% CI 0.47-0.69) or postpartum hemorrhage (RR 0.82, 95\% CI 0.70-0.96). No maternal deaths were reported in either group.

Less than $1 \%$ of infants in both groups had an Apgar score of less than 4 at 5 minutes $(0.1 \%$ in the planned home birth group and $0.2 \%$ in the planned hospital birth group); 28 infants $(0.24 \%)$ in each group required positive pressure ventilation and chest compressions (Table 3). More infants in the home birth group weighed less than $2500 \mathrm{~g}$ (RR 1.41, 95\% CI 1.00-1.97). Infants born to mothers who planned home birth were more likely to be exclusively breastfed at 3 (RR 1.09 , 95\% CI 1.08-1.10) and 10 days postpartum (RR 1.11, 95\% CI 1.10-1.13). We found no difference in our primary composite outcome of stillbirth, neonatal death or morbidity, with an absolute risk of $0.39 \%$ in each group (RR 1.03, 95\% CI 0.68-1.55) (Table 4).

\section{Subgroup analysis}

Among women planning home birth, first-time mothers were less likely than women having subsequent births to actually give birth at home. The risk of the primary outcome was not different between planned home or planned hospital groups among either first-time or subsequent pregnancies (Table 4). Regardless of place of birth, women having their first baby had an increased likelihood of augmented labour, receiving pain relief, having assisted vaginal or cesarean delivery and undergoing episiotomy and perineal trauma; however, both first-time mothers and multiparous mothers planning home birth experienced these outcomes significantly less frequently than women planning hospital births (Table 2). After stratifying by parity, a lower risk of postpartum hemorrhage remained for multiparous mothers who planned a home birth compared with those who planned a hospital birth, but not among first-time mothers. More infants born to first-time mothers planning home birth were less than $2500 \mathrm{~g}$ compared with those planning hospital birth, but this did not hold true for multiparous mothers (Table 3).

\section{Sensitivity analysis}

Inclusion of the records that indicated a home birth was planned at the onset of labour, but which were assessed as being highly likely to reflect a data error, did not result in any difference in the primary composite outcome or any of the component morbidity or mortality outcomes (Appendix 2, available at www.cmaj.ca/lookup/suppl/doi:10 .1503/cmaj.150564///DC1).

\section{Meta-analysis}

Among women included in our 2 studies $(n=$ 18184 planned home births and $n=18181$ planned hospital births), ${ }^{5}$ the incidence of stillbirth or neonatal death excluding lethal anomalies within 28 days was 1.15 for every 1000 births (95\% CI $0.73-1.80)$ planned to be at home and 0.94 for every 1000 births $(95 \% \mathrm{CI}$ 0.56-1.53) planned to be in hospital (RR 1.24, 95\% CI 0.65-2.34). Among first-time mothers (6320 with planned home births and 6324 with planned hospital births), the incidence was 1.90 for every 1000 planned home births $(95 \%$ CI 1.03-3.42) and 1.90 for every 1000 planned hospital births (95\% CI 1.03-3.41) (RR 1.00, 95\% CI 0.45-2.23). Among multiparous mothers (11 858 with planned home births and 11857 with planned hospital births), the incidence was 0.76 for every 1000 planned home births (95\% CI $0.37-1.50$ ) and 0.42 for every 1000 planned hospital births (95\% CI 0.161.05) (RR 1.80, 95\% CI 0.60-5.37).

\section{Interpretation}

Among women who intended to birth at home with midwives in Ontario, the risk of stillbirth, neonatal death or serious neonatal morbidity was low and did not differ from midwifery clients who chose hospital birth. Compared with women who planned to birth in hospital, women 
Table 3: Comparison of neonatal variables in planned home and hospital groups with relative risk presented for selected outcomes

\begin{tabular}{|c|c|c|c|}
\hline \multirow[b]{2}{*}{ Variable } & \multicolumn{2}{|c|}{ No. $(\%) *$} & \multirow[b]{2}{*}{$\operatorname{RR}(95 \% \mathrm{Cl})$} \\
\hline & $\begin{array}{c}\text { Planned home } \\
\quad n=11493 \\
\text { (nulliparous } n=4027 \\
\text { multiparous } n=7466 \text { ) }\end{array}$ & $\begin{array}{c}\text { Planned hospital } \\
\quad n=11493 \\
\text { (nulliparous } n=4027 \\
\text { multiparous } n=7466 \text { ) }\end{array}$ & \\
\hline \multicolumn{4}{|l|}{ Birthweight, g } \\
\hline$<2500$ & $79 \quad(0.7)$ & $56 \quad(0.4)$ & \\
\hline $2500-4000$ & 9253 (80.9) & 9417 (82.3) & \\
\hline$>4000$ & $2101(18.4)$ & 1967 (17.2) & \\
\hline Missing & 60 & 53 & \\
\hline Apgar score < 4 at $1 \mathrm{~min}$ & $136 \quad(1.2)$ & $183 \quad(1.6)$ & \\
\hline Missing & 123 & 68 & \\
\hline Apgar score $<7$ at $5 \mathrm{~min}$ & $86 \quad(0.7)$ & $109 \quad(0.9)$ & \\
\hline Missing & 100 & 74 & \\
\hline \multicolumn{4}{|l|}{ Infant resuscitation } \\
\hline PPV & $553 \quad(4.8)$ & $530 \quad(4.6)$ & \\
\hline PPV and chest compressions & $28 \quad(0.2)$ & $28 \quad(0.2)$ & \\
\hline $\begin{array}{l}\text { Clinically significant congenital } \\
\text { anomalies }\end{array}$ & $241 \quad(2.1)$ & $334 \quad(2.9)$ & \\
\hline Cardiovascular & $41 \quad(0.4)$ & $72 \quad(0.6)$ & \\
\hline Neural tube defects & $3 \quad(0.03)$ & $2 \quad(0.02)$ & \\
\hline Other CNS & $1 \quad(0.01)$ & $4 \quad(0.03)$ & \\
\hline Gastrointestinal & $6 \quad(0.05)$ & $13 \quad(0.1)$ & \\
\hline Facial anomalies & $18 \quad(0.2)$ & $20 \quad(0.2)$ & \\
\hline Genitourinary & $47 \quad(0.4)$ & $66 \quad(0.6)$ & \\
\hline Musculoskeletal & $42 \quad(0.4)$ & $50 \quad(0.4)$ & \\
\hline Respiratory & $4 \quad(0.03)$ & $4 \quad(0.03)$ & \\
\hline Down syndrome & $10 \quad(0.1)$ & $10 \quad(0.1)$ & \\
\hline Other & $77 \quad(0.7)$ & $105 \quad(0.9)$ & \\
\hline Birth weight $<2500 \mathrm{~g}$ & $79 \quad(0.7)$ & $56 \quad(0.5)$ & $1.41(1.00-1.97)$ \\
\hline Nulliparous & $45 \quad(1.1)$ & $26 \quad(0.6)$ & $1.73(1.07-2.80)$ \\
\hline Missing & 22 & 22 & \\
\hline Multiparous & $34 \quad(0.5)$ & $30 \quad(0.4)$ & $1.13(0.70-1.85)$ \\
\hline Missing & 38 & 31 & \\
\hline Exclusive breastfeeding at $3 \mathrm{dt}$ & $10300(90.0)$ & 9446 (82.9) & $1.09(1.08-1.10)$ \\
\hline Nulliparous & $3455(86.2)$ & 3161 (79.3) & $1.09(1.07-1.11)$ \\
\hline Missing & 18 & 38 & \\
\hline Multiparous & $6845(92.1)$ & 6285 (84.9) & $1.09(1.07-1.10)$ \\
\hline Missing & 34 & 60 & \\
\hline Exclusive breastfeeding at $10 \mathrm{df}$ & $9566(87.8)$ & 8459 (78.9) & $1.11(1.10-1.13)$ \\
\hline Nulliparous & $3220(84.8)$ & 2825 (74.9) & $1.13(1.11-1.16)$ \\
\hline Missing & 223 & 250 & \\
\hline Multiparous & $6346(89.6)$ & $5634 \quad(81.2)$ & $1.10(1.09-1.12)$ \\
\hline Missing & 377 & 522 & \\
\hline $\begin{array}{l}\text { Any perinatal/neonatal death } \\
\text { including lethal anomalies }\end{array}$ & $13 \quad(0.1)$ & $13 \quad(0.1)$ & $1.00(0.46-2.16)$ \\
\hline Nulliparous & $7 \quad(0.2)$ & $8 \quad(0.2)$ & $0.86(0.32-2.41)$ \\
\hline Multiparous & $6 \quad(0.1)$ & $5 \quad(0.1)$ & $1.20(0.37-3.93)$ \\
\hline
\end{tabular}


who planned to birth at home underwent fewer obstetrical interventions, were more likely to have a spontaneous vaginal birth and were more likely to be exclusively breastfeeding at 3 and 10 days after delivery. These findings were true for the whole group as well as for the subgroups of first-time and multiparous mothers.

It is reassuring that the risk of death in both groups in our study was comparable to the risk found in other studies of low-risk birth in Can-

Table 4: Primary outcome and component outcomes

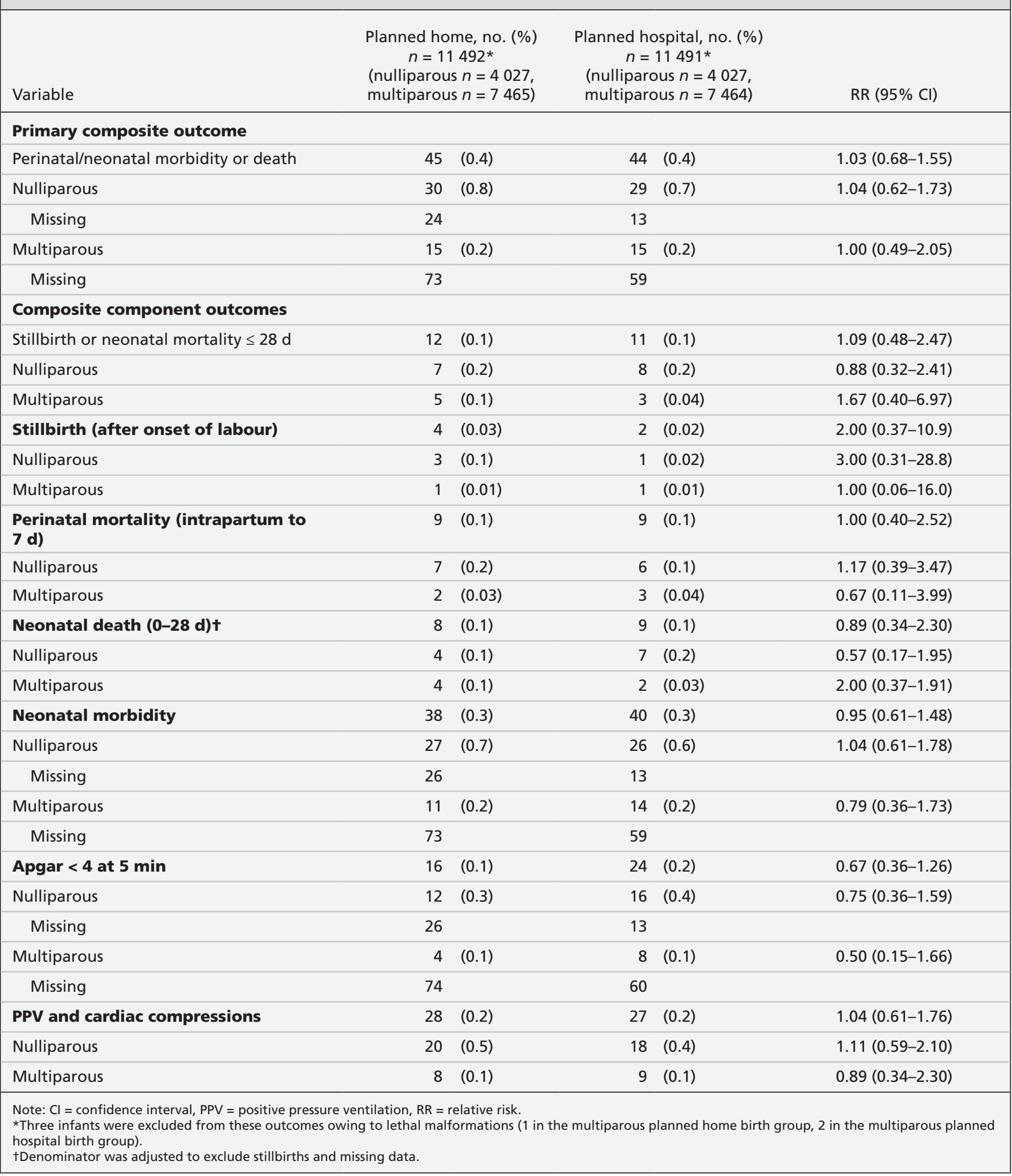


$\operatorname{ada}^{5,7,15}$ and internationally.,16 The UK Birthplace study found a significant increase in the odds of neonatal morbidity or death among firsttime mothers who planned a home birth compared with those who planned to give birth in an obstetric unit. ${ }^{4}$ We did not find a difference in risk of neonatal morbidity or death by planned place of birth among first-time mothers; however, the measures of neonatal morbidity used by the UK Birthplace Study differed considerably from ours. Our findings add further support to the Cochrane review of home births that recommended that health care systems include a wellintegrated home birth service for women with low-risk pregnancies ${ }^{8}$ and to the NICE guidelines that support women receiving information about birth outside of hospital settings. ${ }^{9}$

\section{Strengths and limitations}

Our data set used reports detailing all women who entered midwifery care in Ontario, about one-quarter of whom planned a home birth, which resulted in a reasonable sample size. The data set was complete and provided reasonable detail in terms of labour, birth and neonatal outcomes of interest. The same midwives provided care for women planning both home and hospital births, thus removing the potential confounder of care provider when comparing outcomes. We accounted for parity by matching on nulliparity in the planned home and hospital groups, which provided the outcomes in a format that is useful for pregnant women and their care providers, and is easily included in meta-analyses. We included the meta-analyses of all Ontario planned home births from 2003 to 2009.

Maternal hemorrhage was not defined within the data set, which may lead to considerable variation in reporting. The data set has changed since our earlier study, limiting our ability to report on admission to neonatal intensive care units and duration of stay. The completion of maternal body mass index data was poor (missing for $49 \%$ of records), limiting our ability to examine this factor as a potential confounder. The study reported no maternal deaths; however, with a very low national incidence of 6.1-11.9 for every 100000 births, ${ }^{17}$ much larger studies would be required to have adequate power to address this issue. In total, 130 records were excluded because they were missing values for matching variables (parity or number of previous cesarean deliveries) or exposure classification (planned place of birth). Among the 62 records that would have met all other inclusion criteria, one infant composite outcome occurred. This record was missing planned place of birth and indicated a neonatal death. This missing data was not accounted for in our analyses; however, given our large sample size, the impact that this missing data may have had on our results is likely small.

This study reports on outcomes of planned home birth in a jurisdiction where women are attended by registered midwives whose education and practice includes home birth and who are required by their regulatory college to maintain competence in providing care in the home setting. Home birth is offered as part of midwifery care as an option to women who have undergone screening and is an integrated part of the health care system, which facilitates access to emergency transportation and transfer of care to obstetric or pediatric services when required. Self-selection by women combined with careful screening by midwives likely contributes to positive outcomes. It is possible that even though the same midwives provided care in home and in hospital, there may be unmeasured differences in clinical management in different settings. Therefore, the health care system setting, the unmeasured differences in the care provided and the women choosing their planned place of birth likely contributed to the lower interventions and similar neonatal outcomes associated with planned home births compared with planned hospital births. As more women choose home birth and as the midwifery profession grows in Ontario, it will be interesting to see whether the lower intervention rates that have been consistently observed to date among women who plan home births are sustained.

\section{Conclusion}

Women with low-risk pregnancies who are planning home births in Ontario attended by midwives should not anticipate any increased risk of perinatal morbidity or death compared with planning a hospital birth. These findings extend to both nulliparous and multiparous women in this setting. Findings from the study should be generalized only to settings that provide similar support for women choosing home birth.

\section{References}

1. MacDonald ME, Bourgeault IL. The Ontario midwifery model of care. In: Davis-Floyd RE, Barclay L, Tritton J, Daviss BA, editors. Birth models that work. Oakland (CA): University of California Press; 2009. p. 89-117.

2. Better outcomes registry \& network (BORN) Ontario. Annual Report 2012-2013 and 2013-2014. Ottawa: BORN Ontario; 2015. Available: https://www.bornontario.ca/assets/documents/special reports/121187 Final Web - English.pdf (accessed 2015 Nov. 25).

3. Olsen O, Clausen JA. Planned hospital birth versus planned home birth. Cochrane Database Syst Rev 2012;(9):CD000352.

4. Brocklehurst P. Perinatal and maternal outcomes by planned place of birth for healthy women with low risk pregnancies: the Birthplace in England national prospective cohort study. BMJ 2013;344:17. 
5. Hutton EK, Reitsma AH, Kaufman K. Outcomes associated with planned home and planned hospital births in low-risk women attended by midwives in Ontario, Canada, 2003-2006: a retrospective cohort study. Birth 2009;36:180-9.

6. Janssen Patricia A, Lee Shoo K, Ryan Elizabeth M, et al. Outcomes of planned home births versus planned hospital births after regulation of midwifery in British Columbia. CMAJ 2002;166:315-23.

7. Janssen PA, Saxell L, Page LA, et al. Outcomes of planned home birth with registered midwife versus planned hospital birth with midwife or physician. CMAJ 2009;181:377-83.

8. Ole O, Clausen Jette A. Planned hospital birth versus planned home birth. Cochrane Database Syst Rev 2000;(2)CD000352.

9. Intrapartum care: care of healthy women and their babies during childbirth. London (UK): Royal College of Obstetricians and Gynaecologists;2014. Available: www.nice.org.uk/guidance/ CG190 (accessed 2015 Nov. 17).

10. Indications for mandatory discussion, consultation and transfer of Care. Toronto: College of Midwives of Ontario; 2000. Available: www.cmo.on.ca/wp-content/uploads/2015/07/ FINAL-CONSULTATION-AND-TRANSFER-OF-CARE.pdf (accessed 2015 Nov. 25).

11. Vaginal birth after cesarean section and choice of birthplace. Toronto: College of Midwives of Ontario; 2014. Available: www. cmo.on.ca/wp-content/uploads/2015/07/FINAL-Vaginal-Birth -After-Cesarean-Section-and-Choice-of-Birthplace-May-2014.pdf (accessed 2015 Nov. 25).

12. Scott JR. Intrapartum management of trial of labour after caesarean delivery: evidence and experience. BJOG 2014;121:157-62.

13. IBM SPSS Statistics for Windows, Version 22.0. Armonk (NY): IBM Corp; 2013.

14. R: A language and environment for statistical computing. Vienna: R Foundation for Statistical Computing; 2011. Available: www.R-project.org/ (accessed 2015 Nov. 17).

15. Janssen PA, Lee SK, Ryan EM, et al. Outcomes of planned home births versus planned hospital births after regulation of midwifery in British Columbia. CMAJ 2002;166:315-23.
16. Jonge de A, van der G BY, Ravelli ACJ, et al. Perinatal mortality and morbidity in a nationwide cohort of 529,688 low-risk planned home and hospital births. BJOG 2009;116:1177-84.

17. Perinatal health indicators for Canada 2013: a report of the Canadian Perinatal Surveillance System. Ottawa: Public Health Agency of Canada; 2013.

Affiliations: Department of Obstetrics and Gynecology (Hutton, Ahmed), The Michael G. DeGroote School of Medicine; Midwifery Education Program (Hutton, Cappelletti, Reitsma, Simioni, Horne, McGregor), Faculty of Health Sciences, McMaster University, Hamilton, Ont.

Contributors: Eileen Hutton and Angela Reitsma were responsible for the study's conception and design. Eileen Hutton, Julia Simioni, Adriana Cappelletti, Jordyn Horne, Caroline McGregor and Rashid Ahmed were responsible for data preparation and statistical analysis. Eileen Hutton, Adriana Cappelletti and Julia Simioni drafted the manuscript. Eileen Hutton, Adriana Cappelletti, Angela Reitsma, Julia Simioni, Jordyn Horne, Caroline McGregor and Rashid Ahmed critically revised the manuscript for important intellectual content. All of the authors approved the final version to be published and agreed to act as guarantors of the work.

Funding: Funding for the analysis of the data in this study was facilitated by an open research grant from the Association of Ontario Midwives. The Association of Ontario Midwives had no role in the design, conduct or interpretation of the analyses presented in this report.

Data sharing: No additional data are available. 